Von der Transmission bis zur Therapie

Hepatitis A und

Hepatitis E - Epidemiologie und natürlicher Verlauf

\author{
J. Hadem, H. Wedemeyer \\ Abeilung Gastroenterologie, Hepatologie und Endokrinologie, \\ Medizinische Hochschule Hannover \\ (Direktor: Prof. Dr. M.P. Manns)
}

klinikarzt 2005; 34 (5): 123-127

S owohl die Hepatitis A als auch die Hepatitis E sind reiseassoziierte Infektionen, die oral oder fäkal übertragen werden - häufig ist die Infektion Folge schlechter Hygieneverhältnisse, einer schlechten Trinkwasser- und Nahrungsmittelqualität, einer fehlenden Abwasserkanalisation oder einer hohen Bevölkerungsdichte.

\section{Hepatitis A}

Epidemiologie und Transmission

Die Hepatitis A ist weltweit die häufigste Ursache akuter viraler Hepatitiden. Der Erreger, das HepatitisA-Virus (HAV), ist ein kleines, hepatotropes, positiv-strängiges RNAVirus des Genus Hepatovirus der Picornaviridae. Man kann Gebiete mit hoher, intermediärer und niedriger HAV-Prävalenz unterscheiden (Abb. 1).

Die Weltgesundheitsorganisation schätzt die jährliche Zahl von Hepatitis-A-Erkrankungen auf 1,4 Millionen. Dabei scheint es zyklische Schwankungen mit einem Ansteigen der Inzidenz etwa alle zehn Jahre zu geben. Im Zeitraum von 1980 bis 2001 wurden im Durchschnitt 25000 Erkrankungsfälle an das Center for Disease Control (CDC) gemeldet (6). Die Zahl der tatsächlichen Neuinfektionen in den USA dürfte jedoch mindestens zehnfach höher

Das Hepatitis-A-Virus (HAV), das als Hepatovirus in die Familie der Picornaviren gehört, ist die häufigste Ursache viraler Hepatitiden weltweit. Die oral-fäkale Transmission des Hepatitis-AVirus ist häufig Folge suboptimaler Hygieneverhältnisse, schlechter Trinkwasser- und Nahrungsmittelqualität, fehlender Kanalisation von Abwässern und einer hohen Bevölkerungsdichte. In Deutschland treten Hepatitis-A-Erkrankungen häufig reiseassoziiert auf, die Prävalenzraten gehen seit einigen Jahren stetig zurück. Die abnehmende Inzidenz der Hepatitis-AInfektion auch bei Kindern und Jugendlichen in den westlichen Ländern erhöht das Risiko symptomatischer akuter HAV-Infektionen im Erwachsenenalter, die in Einzelfällen - wenn zusätzliche Risikofaktoren vorliegen - schwer oder sogar lebensbedrohlich verlaufen können. Daher sollten insbesondere Patienten mit vorbestehender Lebererkrankung gegen Hepatitis A geimpft werden. Vor allem in den (sub-)tropischen Regionen Asiens spielen Infektionen mit dem Hepatitis-E-Virus (HEV), dessen taxonomische Klassifikation noch unklar ist, eine Rolle. Auch diese Infektionen werden oral-fäkal übertragen. Wahrscheinlich erfolgt der Großteil der humanen Hepatitis-E-Infektionen durch die Kontamination von Nahrungsmitteln oder Trinkwasser über Nutz- und Wildtiere. Im Jahr 2003 wurden dem Robert-Koch-Institut 32 Hepatitis-E-Infektionen gemeldet. Auch hier waren die meisten Erkrankungsfälle mit einem Aufenthalt in Endemiegebieten assoziiert.

liegen - etwa bei 263000 HAV-Infektionen jährlich (4).

Daten aus dem Robert-Koch-Institut (RKI) zeigen für Deutschland einen deutlichen Rückgang der Hepatitis-A-Inzidenz seit 1980 (Abb. 2). Während im Jahr 20012272 Hepatitis-A-Fälle an das RKI übermittelt wurden, waren dies im Jahr 2003 nur noch 1365 Fälle. Die Inzidenz der Hepatitis A betrug somit 2001 im Durchschnitt bundesweit 2,75 pro 100000 Einwohner und 1,7 pro 100000 Einwohner im Jahr 2003. Allerdings bestehen zum Teil deutliche regionale Schwankungen mit niedrigeren Werten für ländliche Gebiete und Häufungen in Ballungszentren. In Hamburg beispielsweise betrug die HAV-Inzidenz im Jahr 2003 acht pro 100000 Einwohner (17).

Die unterschiedliche Verteilung der weltweiten HAV-Prävalenz erklärt sich durch diskrepante Hygienestandards. In den Entwicklungsländern erfolgt die asymptomatische oder klinisch milde Infektion in der Regel bereits im Kindesalter, sodass hier mehr als 70\% der Bevölkerung gegen das Hepatitis-A-Virus (HAV) immun sind. Dahingegen haben verbesserte Hygienestandards und die Einführung der Hepatitis-A-Vakzinierung Mitte der 90er Jahre in den 


\section{Abb. 1 Geografische Verteilung der Hepatitis-A-Prävalenz im Jahr 2002}

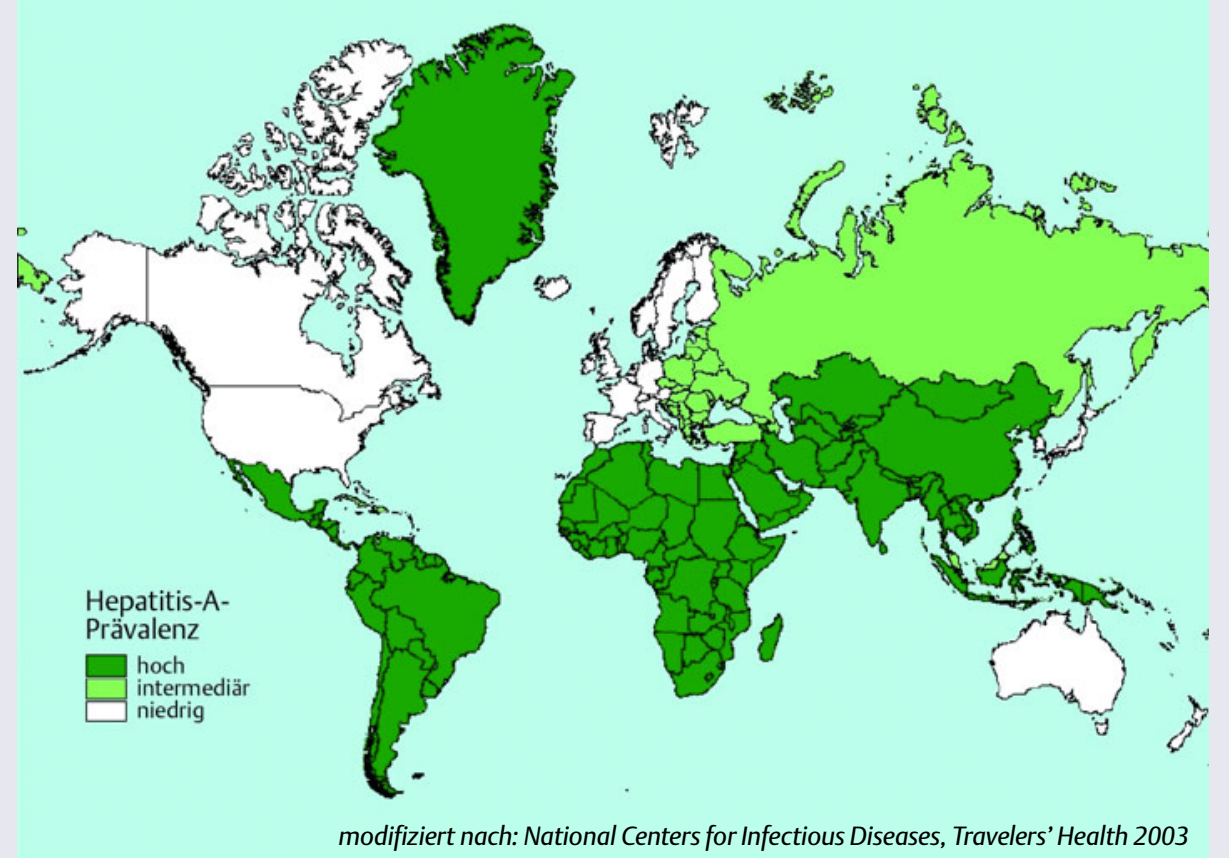

Bei Reisen in Länder mit hoher ( $\geq 20$ Erkrankungsfälle/100 000 Einwohner) oder intermediärer Hepatitis-A-Virus-Prävalenz (10-19 Erkrankungsfälle/100 000 Einwohner) ist eine Impfung empfehlenswert

industrialisierten Ländern zu einem Rückgang der HAV-Infektionen im Kindesalter geführt, nur etwa $2 \%$ der Kinder zwischen 5 und 14 Jahren sind hier mittlerweile seropositiv (7). Die Seropositivität der Gesamtbevölkerung in den westlichen Industrienationen dürfte um $30 \%$ liegen (12).

Demzufolge sind immer mehr Urlauber durch eine Infektion mit dem Hepatitis-A-Virus im Erwachsenenalter gefährdet. Und im Gegensatz zu Kindern, bei denen diese Infektion meist asymptomatisch verläuft, entwickeln etwa $75 \%$ der Erwachsenen eine symptomatische

\section{Tab. 1 Hygienemaßnahmen zur Vor-} beugung einer Hepatitis $A$ und $E$

- Obst waschen bzw. schälen

- rohe Salate und ungekochte Meeresfrüchte meiden

- Leitungswasser und Eiswürfel meiden (auch nicht zum Zähneputzen!)

- Wasser abkochen (> 3 min) oder chemisch sterilisieren (Handfilter bieten keinen Schutz)

- öffentliche sanitäre Einrichtungen meiden

- gegebenenfalls alkoholische Händedesinfektion durchführen

- generell gilt: „Cook it, peel it or forget it!“ akute Hepatitis, die in Einzelfällen zumal wenn zusätzliche Risikofaktoren vorhanden sind - schwer bis lebensbedrohlich sein kann (13). Die Krankenhausstatistik des Statistischen Bundesamtes vermerkt für das Jahr 2001 insgesamt 1666 aus dem Krankenhaus entlassene vollstationäre Patienten mit einer durchschnittlichen Liegezeit von 8,8 Tagen. Laut Todesursachenstatistik kommt es in Deutschland jährlich zu 9-19 HAV-assoziierten Todesfällen (17).

Bei etwa der Hälfte aller Erkrankten lässt sich die Infektionsquelle eruieren. Dabei ist eine Hepatitis-A-Erkrankung im familiären Umfeld der bedeutendste Risikofaktor für eine Hepatitis-A-Neuinfektion. In selteneren Fällen erfolgt die Transmission über HAV-kontaminierte Nahrungsmittel, wobei es zu Hepatitis-A-Ausbrüchen mit mehreren Neuerkrankten kommen kann. Solche Vorfälle sind in Entwicklungsländern aufgrund der hohen Seroprävalenz selten.

Nur etwa $60 \%$ der Hepatitis-AFälle in Deutschland, die nach dem Infektionsschutzgesetz (IfSG) gemeldet werden, wurden in Deutsch- land erworben (17). Mit einer Reise in ein HAV-Endemiegebiet waren 33\% aller dokumentierten Fälle assoziiert, $10 \%$ wurden am ehesten durch einen intravenösen Drogenkonsum erworben und $8 \%$ traten im Umfeld von Kindergarten und Schule auf. Bei der ausländischen Wohnbevölkerung war die Erkrankung meist auf den Import der Infektion durch Kinder zurückzuführen, ein Großteil dieser Infektionen fand in der Türkei statt (3).

Infizierte Personen scheiden das Hepatitis-A-Virus mit dem Fäzes aus. Die Übertragung erfolgt entweder durch einen direkten Personenkontakt oder durch die Exposition mit kontaminiertem Wasser, Eis, Fisch, Früchten, Gemüse oder anderen Speisen, die während der Ernte oder der Weiterverarbeitung mit dem Virus verunreinigt und anschließend ungekocht gegessen wurden. Das Risiko einer HepatitisA-Virus-Infektion variiert mit den Lebensbedingungen, der Dauer des Auslandsaufenthaltes und der Prävalenz im Zielland. Reisende nach Nordamerika, Japan, Australien, Neuseeland und Westeuropa haben kein erhöhtes Risiko zu erwarten (19). Dagegen ist das Hepatitis-ARisiko bei Reisen nach Italien, dem angrenzenden Mittelmeerraum und Osteuropa etwa dreifach und bei Reisen nach Asien, Afrika und Lateinamerika neunfach erhöht.

Besonders gefährdet sind Urlauber in ländlichen Gebieten, die regelmäßig unter suboptimalen hygienischen Verhältnissen Nahrung zu sich nehmen. Das Risiko steigt hier im Vergleich zu einer Hotelunterkunft um das Siebenfache. Dennoch kann die Hepatitis A durchaus auch in gehobenen Unterkünften auftreten. Daher sollte im Zweifelsfall Leitungswasser weder getrunken noch zum Zähneputzen verwendet, und Eiswürfel sollten gemieden werden. Risikoreich ist auch der Genuss von rohen Salaten, ungeschältem Obst und ungekochten Meeresfrüchten (8). Einfache Regeln für den Verzehr von Speisen in Risikogebieten sind in der Tabelle 1 kurz zusammengefasst. Generell gilt beim Verzehr von Speisen in Endemiegebieten: „Cook it, peel it or forget it“. 
HAV-Infektionen im Zusammenhang mit einem intravenösen Drogenkonsum sind ebenfalls beschrieben worden. Einige Studien legen zudem nahe, dass das Risiko einer Hepatitis-A-Infektion bei homosexuell aktiven Männern erhöht ist. Dennoch scheint der Kontakt mit Samenflüssigkeit oder Vaginalsekret keine bedeutende Rolle bei der HAVTransmission zu spielen.

Diagnose und natürlicher Verlauf

Serologische Tests ermöglichen die Abgrenzung der Hepatitis A von anderen viralen Hepatitiden. AntiHAV-IgM ist bereits fünf bis zehn Tage vor Beginn der Symptome im Serum nachzuweisen und fällt dann innerhalb von sechs Monaten unter die Nachweisgrenze ab (Abb. 3). Die Sensitivität und Spezifität heutiger anti-HAV-IgM-Assays liegt bei über 95\%. Gesamt-anti-HAV (IgM + IgG) ist zum Zeitpunkt der klinischen Manifestation und auch darüber hinaus nachweisbar und ist daher ein zuverlässiger Marker für eine eventuell bestehende Immunität gegen das Hepatitis-A-Virus (6). HAVRNA lässt sich in Serum und Stuhl durch eine PCR („polymeraase chain reaction“) nachweisen. Zwar ist eine HAV-PCR zur Sicherung der Diagnose nicht notwendig, sie kann jedoch zur Evaluierung der Infektiosität des Patienten im stationären Alltag hilfreich sein.

Nach der rezeptorvermittelten Internalisation des Hepatitis-A-Virus im Gastrointestinaltrakt kommt es zur Aktivierung angeborener (natürliche Killerzellen, Makrophagen) und erworbener (HLA-restringierte antigenspezifische T-Lymphozyten) Immunmechanismen, welche für die hepatische Inflammation verantwortlich sind (9). Die mediane Inkubationszeit beträgt 28 (15-50) Tage. Dabei besteht die größte Infektiosität in den 14 Tagen vor Auftreten des Ikterus und reduziert sich eine Woche danach wieder.

Im Kindesalter verläuft die Infektion mit Hepatitis-A-Viren regelmäßig asymptomatisch. Hingegen zeigen mehr als 75\% aller jungen Erwachsenen Symptome, die vom milden, anikterischen Krankheitsbild bis hin zur fulminanten Hepatitis reichen können. Die Erkrankung beginnt mit Übelkeit, Bauchschmerz, Fieber, Abgeschlagenheit, dunklem Urin und Ikterus (6). Bei der klinischen Untersuchung findet man typischerweise ein druckschmerzhaftes Abdomen mit Hepatosplenomegalie.

Obwohl sich die meisten Patienten komplett und ohne Residuen von der Erkrankung erholen, wird die Gefahr der Hepatitis A im Erwachsenenalter unterschätzt: Bei etwa $10 \%$ der Patienten nimmt die Erkrankung einen protrahierten, sechs bis neun Monate dauernden Verlauf. Länger persistierende Virämien sind selten. Gravierende Komplikationen bestehen in der Entwicklung von Gerinnungsstörungen, Enzephalopathie und Nierenversagen, sodass bei Patienten ab dem 50 . Lebensjahr von einer Mortalität von $1,8 \%$ auszugehen ist (3). Etwa $4 \%$ aller Fälle von akutem Leberversagen dürften laut einer Studie aus den USA auf eine fulminante Hepatitis A zurückzuführen sein (15).

Die Therapie der akuten Hepatitis A ist rein symptomatisch. Eine antivirale Behandlung mit Interferonen, zum Beispiel analog zur akuten Hepatitis C, ist bei der Hepatitis A nicht indiziert. Insbesondere bei äl-

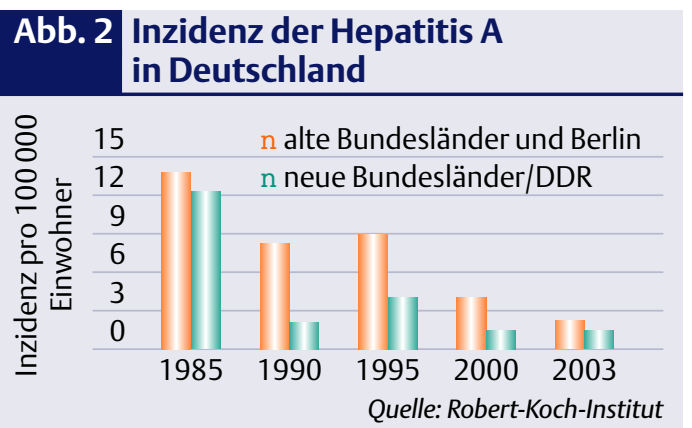

In den letzten Jahren ging die Inzidenz der Hepatitis-A-Infektionen in Deutschland - sowohl in den alten als auch in den neuen Bundesländern kontinuierlich zurück

teren Patienten sind neben den Aminotransferasen die Leberfunktionsparameter engmaschig (je nach Kinetik z.B. zweimal wöchentlich) zu bestimmen. Sollte hier eine signifikante Einschränkung zu verzeichnen sein (Richtwert: Quick < 35\%), ist frühzeitig Kontakt mit einem Transplantationszentrum aufzunehmen.

Seit Mitte der 1990er Jahre stehen monovalente und Kombinationsimpfstoffe gegen Hepatitis A zur Verfügung, die mit Formalin inaktivierte Viruspartikel enthalten und bezüglich des Impferfolges hocheffizient sind. Indiziert ist eine aktive Impfung gegen das Hepatitis-A-Virus

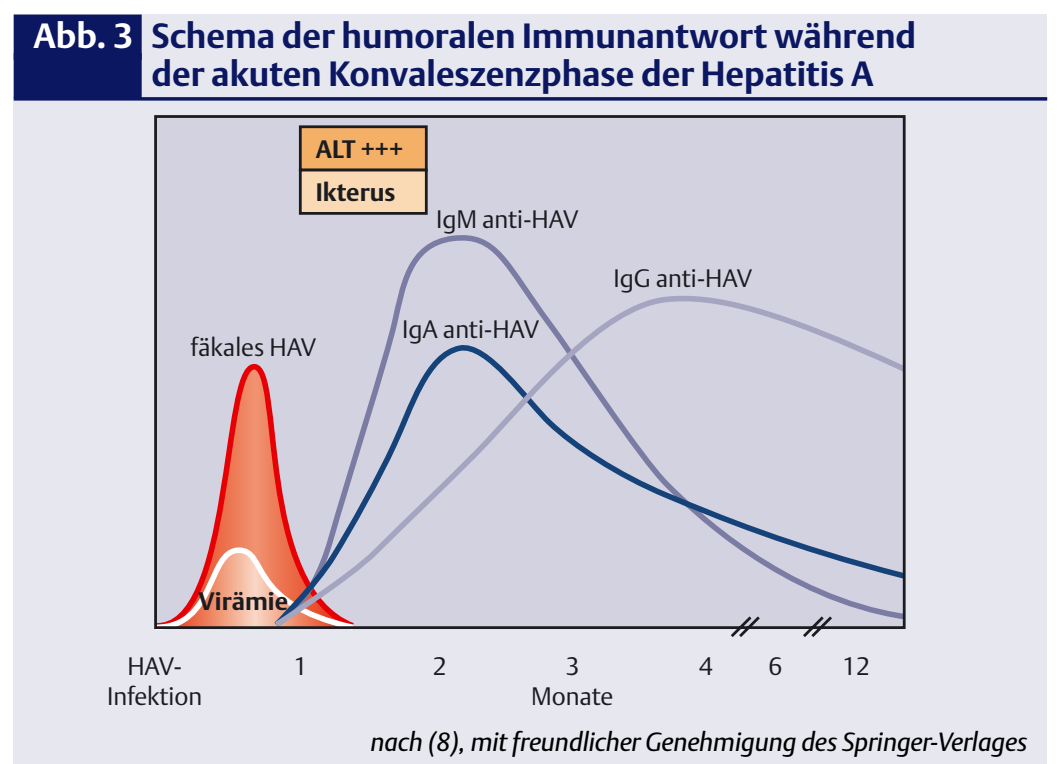

Die fäkale Ausscheidung von Hepatitis-A-Viren sistiert meist 30 Tage nach Beginn der Infektion. Anti-HAV-IgM-Titer erreichen ihr Maximum noch während der Akut- und frühen Konvaleszenzphase und sind nach sechs Monaten bei 75\% der Patienten nicht mehr nachzuweisen. Anti-HAV-IgG persistiert hingegen meist lebenslang 


\section{Abb. 4 Serologische Parameter im Verlauf der akuten Hepatitis E}

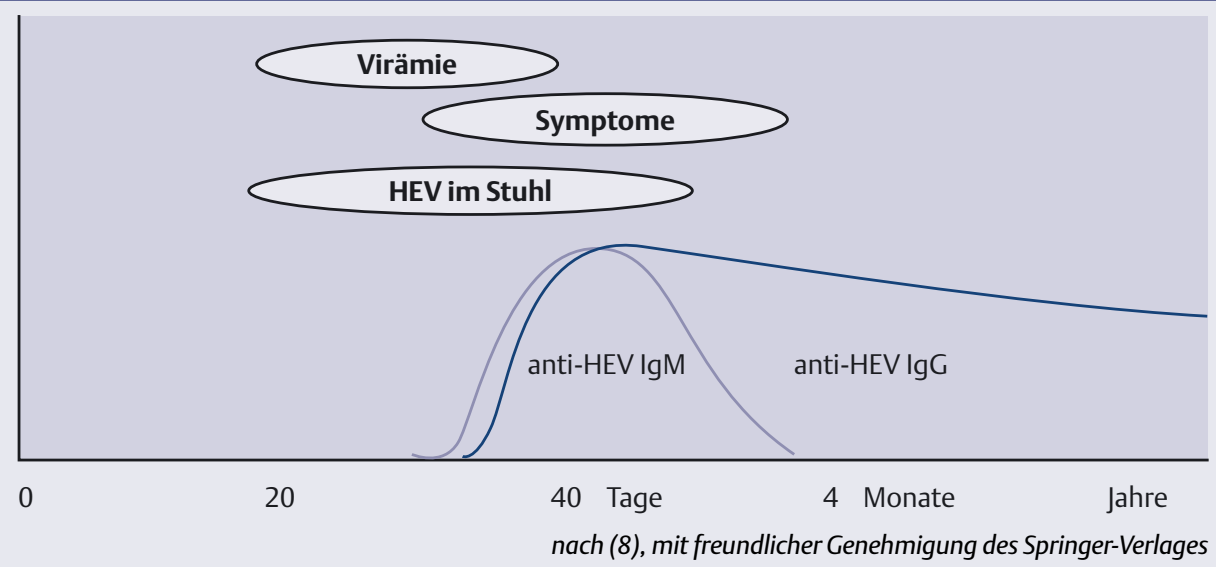

Mit Beginn von Symptomen ist anti-HEV-IgM im Serum nachweisbar. Bereits 14 Tage vorher beginnt die fäkale Ausscheidung des Hepatitis-C-Virus. HEV-RNA ist mittels PCR in Stuhl und Serum nachweisbar

mit einem der genannten inaktivierten Impfstoffe bei Personen ab dem zweiten Lebensjahr vor Reisen in Gebiete mit mittlerer oder hoher HAVPrävalenz (Abb. 1). Besonders zu empfehlen ist eine Impfung dann, wenn zusätzlich zur Reise in ein Endemiegebiet weitere Risikofaktoren vorliegen. Dazu zählen Homosexualität, eine vorbekannte chronische Lebererkrankung, Drogenkonsum, Dialysepflichtigkeit und die regelmäßige Gabe von gepoolten Gerinnungsprodukten $(1,5)$.

Um eine unnötige Impfung $\mathrm{zu}$ vermeiden, sollte eine anti-HAV-IgGTestung bei allen Personen ab dem 40. Lebensjahr und solchen, die aus Regionen mittlerer bis hoher HAVPrävalenz stammen, durchgeführt werden - vorausgesetzt, sie verzögert die rechtzeitige Immunisierung nicht. Bei immunkompetenten Personen ist nach kompletter Impfung keine antiHAV-Titerkontrolle notwendig, die Serokonversionsraten liegen bei über 98\%. Auch eine beschleunigte Impfung (Tag 0, 7, 21 und Monat 12 für Twinrix) ist möglich und führte in einer deutschen Studie zur Induktion von anti-HAV-Antikörpern bei allen gesunden Probanden (11). Verdacht, Erkrankung und Tod an akuter Virushepatitis A sind meldepflichtig.

\section{Hepatitis E}

Epidemiologie und Transmission 1980 wurde das Hepatitis-E-Virus (HEV) als fünftes humanpatho- genes Hepatitisvirus identifiziert. Seine taxonomische Klassifikation ist derzeit noch nicht abschließend geklärt. Einiges spricht dagegen, dass das kleine, unbehüllte, $32 \mathrm{~nm}$ messende RNA-Virus - wie bis vor kurzem angenommen - zur Familie der Caliciviren gehört (16). Epidemien und sporadische Erkrankungsfälle sind aus Afghanistan, Bangladesch, Burma, China, Indien, Indonesien, Kasachstan, Kirgisien, Malaysien, der Mongolei, Nepal, Pakistan, Tajikistan, Turkmenistan, Usbekistan, Mexiko, dem Mittleren Osten, Nord- und Zentralafrika berichtet worden. Dabei scheinen große Epidemien in Zehn-Jahres-Zyklen aufzutreten. Zu den bedeutendsten Epidemien zählten die in Neu Delhi (Indien, 1955 mit 29000 Fällen) und eine in Xinjiang (China, 1958 mit 100000 Fällen) (18).

Im Gegensatz zur Hepatitis A tritt die Hepatitis E hauptsächlich in (sub-)tropischen Entwicklungsländern auf und manifestiert sich generell in älteren Kindern und jungen Erwachsenen. Während in Indien 10-40\% der Erwachsenen über 25 Jahre HEV-Antikörper aufweisen, beträgt die HEV-Seropositivität in den USA und Nordeuropa in dieser Altersgruppe nur etwa 1\%. Im Jahr 2002 wurden dem Robert-Koch-Institut insgesamt 17 Hepatitis-E-Erkrankungen übermittelt, im Jahr 2003 waren es 32 Fälle (Inzidenz 0,04 pro 100000 Einwohner). Die meisten Erkrankungsfälle waren mit einem Aufenthalt in Endemiegebieten assoziiert, bei einigen Patienten blieb die Herkunft allerdings unklar. Im Jahr 2003 erkrankte im Landkreis Marburg-Biedenkopf ein 52-jähriger Mann an Hepatitis E, drei seiner Arbeitskollegen zeigten positive HEVIgG-Titer. Epidemiologische Nachforschungen legten nahe, dass die Infektion über kontaminierte Nahrungsmittel erworben wurde (3).

HEV wird fäkal-oral, hauptsächlich durch kontaminiertes Trinkwasser oder den Verzehr von infiziertem Fleisch übertragen. Ähnlich wie bei der Hepatitis A gelten inadäquate hygienische Verhältnisse als Hauptrisikofaktor für eine Hepatitis-E-Infektion. Das Infektionsrisiko ist für Familienangehörige eines an HepatitisE-Erkrankten aber deutlich niedriger als im Falle einer Hepatitis-A-Infektion (15). Einiges spricht dafür, dass die Hepatitis-E-Infektion - zumindest in einigen Gegenden - eine Zoonose darstellt. So konnten HepatitisE-Viren bzw. HEV-verwandte Viren in Schweinen, Affen, Rehen, Ratten, Mäusen und Schafen nachgewiesen werden. Aufsehen erregt hat ein Bericht aus Japan, bei dem die Übertragung des Virus durch den Verzehr von rohem Rotwildfleisch auf Menschen nachgewiesen wurde (16).

\section{Natürlicher Verlauf}

Müdigkeit, Appetitverlust, Übelkeit, Bauchschmerzen und Fieber sind die Symptome der symptomatischen akuten Hepatitis E, die nach einer Inkubationszeit von 40 (1560) Tagen auftreten. Nach einem Anstieg der Aminotransferasen kommt es zum Ikterus und zu einer Verfärbung des Urins. In den meisten Fällen klingen die Symptome jedoch innerhalb von sechs Wochen ab.

Verglichen mit der Hepatitis A ist die Häufigkeit cholestatischer Verläufe bei der Hepatitis E mit 20-25\% höher (14). Die Rate fataler Ausgänge im Gesamtkollektiv der Erkrankten dürfte weniger als $4 \%$ betragen, wobei ein Alter über 40 Jahre, Bilirubin über einem Wert von $255 \mu \mathrm{mol} / 1$ und eine deutliche Erhöhung der Thromboplastinzeit (INR) als negative prädiktive Faktoren gelten können. Das höchste Ri- 
siko für die Entwicklung einer fulminanten Hepatitis und eines fetalen Abortes scheint hingegen bei einer Infektion in der Schwangerschaft (besonders im zweiten und dritten Trimester) zu bestehen. Hier sind Mortalitätsraten zwischen 15 und $25 \%$ berichtet worden, ohne dass die Pathogenese dieser Krankheitsverläufe aufgeklärt werden konnte (18).

Die akute Hepatitis E heilt bei immunkompetenten Personen aus. Aus Regensburg ist allerdings kürzlich ein erster Fall einer persistierenden Hepatitis-E-Virämie berichtet worden. Hier war das Virus bei einem nierentransplantierten Patienten mit unklarer Hepatitis über mehr als zwei Jahre nachzuweisen (2). Bei unklaren Hepatitiden sollte eine Hepatitis E unbedingt bei der Differenzialdiagnose berücksichtigt werden.

Die Diagnose einer Hepatitis E erfolgt durch Nachweis von antiHEV (IgM und IgG), welches bereits mit Beginn der klinischen Symptomatik nachweisbar ist (Abb. 4). Zusätzlich kann HEV-RNA mittels PCR zu Beginn der Erkrankung im Stuhl und häufig auch im Serum nachgewiesen werden.

\section{From Transmission to Therapy - Hepatitis A and Hepatitis E:} Epidemiology and Natural Course of the Disease

Hepatitis A virus (HAV) belongs to the hepatovirus genus of the picornavirus family and is the most common defined cause of viral hepatitis worldwide. HAV infection is usually acquired via the fecal-oral route, and person-to-person spread is enhanced under poor hygienic conditions and overcrowding causing contamination of food and water supplies. Hepatitis A in Germany is largely associated with travel into countries with intermediate or high hepatitis A virus prevalence. According to observations of the German Robert-Koch-Institut, HAV prevalence in Germany has markedly decreased from 1980 to now with 1365 registered hepatitis $A$ cases in 2003. As immunity to HAV in german children and young adults decreases, there is a growing subpopulation at risk for HAV infection that might be associated with a severe course of disease in older individuals. Therefore, vaccination is recommended in persons at high risk for HAV infection - among them preexisting liver disease and travel to intermediate or high prevalence countries. Hepatitis E, which is presently unclassified, is endemic in the (sub-)tropical regions of asia. Like in case of hepatitis $A$, its transmission follows a fecal-oral route. But in contrast to HAV, many human infections are acquired directly or via contamination of water from a variety of domestic and wild animals. In 2003, 32 cases of hepatitis $E$ virus infection were registered at the Robert-KochInstitut, most of them in association with travel into a high prevalence country.

\section{Key Words}

hepatitis A - hepatitis E - epidemiology - transmission - natural course

\section{Literatur bei der Redaktion / im Internet unter www.klinikarzt.info}

\section{Anschrift für die Verfasser}

Dr. Johannes Hadem

Abteilung Gastroenterologie, Hepatologie und Endokrinologie

Medizinische Hochschule Hannover

Carl-Neuberg-Str. 1

30625 Hannover 